\title{
How flat is an air-cleaved mica surface?
}

\author{
F Ostendorf ${ }^{1}$, C Schmitz ${ }^{1}$, S Hirth ${ }^{1}$, A Kühnle ${ }^{1}$, J J Kolodziej ${ }^{2}$ and \\ M Reichling ${ }^{1}$
}

\author{
${ }^{1}$ Fachbereich Physik, Universität Osnabrück, Barbarastraße 7, 49076 Osnabrück, Germany \\ ${ }^{2}$ Research Centre for Nanometer-Scale Science and Advanced Materials (NANOSAM), \\ Faculty of Physics, Astronomy and Computer Science, Jagiellonian University, \\ ulica Reymonta 4, 30-059 Krakow, Poland \\ E-mail: kuehnle@uos.de
}

Received 17 April 2008, in final form 20 April 2008

Published 12 June 2008

Online at stacks.iop.org/Nano/19/305705

\begin{abstract}
Muscovite mica is an important mineral that has become a standard substrate, due to its easy cleavage along the $\{001\}$ planes, revealing a very flat surface that is compatible with many biological materials. Here we study mica surfaces by dynamic atomic force microscopy (AFM) operated in the non-contact mode (NC-AFM) under ultra-high vacuum (UHV) conditions. Surfaces produced by cleaving in UHV cannot be imaged with NC-AFM due to large surface charges; however, cleavage in air yields much less surface charge and allows for NC-AFM imaging. We present highly resolved NC-AFM images of air-cleaved mica surfaces revealing a rough morphology originating from a high density of nanometre-sized particles. Among these particles, we find regularly shaped structures indicating the growth of crystallites on the surface. The contamination layer cannot be removed by degassing in UHV; even prolonged heating at a temperature of $560 \mathrm{~K}$ under UHV conditions does not yield an atomically flat surface.
\end{abstract}

(Some figures in this article are in colour only in the electronic version)

\section{Introduction}

Mineral surfaces are abundant in nature. They have diverse functions in the non-living world as well as for living organisms, and play a key role in fundamental research on mineral dissolution, precipitation, and crystal growth $[1,2]$. One of the most prominent mineral surfaces is that of muscovite mica, which is renowned for its very large, atomically flat, and chemically inert surfaces produced by perfect cleavage parallel to the $\{001\}$ planes [3]. Mica is widely used as a substrate in fields such as scanning probe microscopy, biotechnology, and material science, because its perfect cleavage makes it suitable for depositing biological materials $[4,5]$, thin metallic films [6, 7], or ordered molecular layers $[8,9]$. To interpret and predict the adsorption and possibly reaction of these materials on the mica surface, it is, therefore, of greatest importance to understand the structure and morphology of cleaved mica surfaces down to the atomic scale.

It has long been known that air-cleaved mica surfaces are covered with a layer of airborne contaminants [10, 11] which readily adsorb on freshly cleaved faces. The process of surface degradation is facilitated by surface charges, and it has been proposed that specifically carbonaceous gases react with water present at the surface [3]. Studies with the surface force apparatus indicate that these surface precipitates are water soluble and that the contamination layer can be removed by immersing the mica surface in water [11-13]. When using mica as a substrate in UHV applications, the mica surfaces are usually cleaved in air, transferred into the UHV chamber, and degassed under UHV conditions [6-9, 14]. It is generally believed that annealing mica surfaces for $1-2 \mathrm{~h}$ under UHV conditions at temperatures around $500 \mathrm{~K}$ removes the adsorbed water film and other contaminants that are present on the surface, revealing an atomically flat surface. In this contribution we demonstrate that this is not at all the case, and we shed light on the apparent surface structure of mica subject to thermal treatment in UHV.

Muscovite mica belongs to the family of clay minerals, and the crystal structure of mica is typically associated with the chemical formula $\mathrm{KAl}_{2}\left(\mathrm{Si}_{3} \mathrm{Al}\right) \mathrm{O}_{10}(\mathrm{OH})_{2}$. A bulk crystal of mica is composed of negatively charged aluminosilicate layers in which the negative charge of the layers arises from a substitution of a quarter of the $\mathrm{Si}^{4+}$ ions by $\mathrm{Al}^{3+}$ ions. These layers are kept together by electrostatically bound interlayer cations, namely potassium ions $\left(\mathrm{K}^{+}\right)$, compensating the charge, as shown in the model structure of figure 1(a), which is a projection of the crystal onto the $a$-axis. The easy 

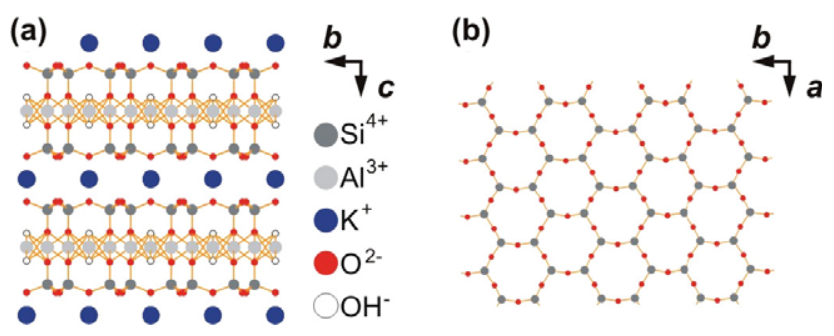

Figure 1. Schematic representation of the muscovite mica crystal structure. Vectors $a$ and $b$ define the $\{001\}$ planes, vector $c$ is the surface normal vector. (a) Side-view (projection onto the $a$-axis) exhibiting aluminosilicate layers separated by electrostatically bound interlayer potassium ions. (b) Hexagonal arrangement of the $\{001\}$ surface top layer (projection onto the $c$-axis) exhibiting $\mathrm{Si}$ (partly $\mathrm{Al}$ ) and $\mathrm{O}$ atoms of a cleaved mica surface, residual potassium ions are not displayed.

cleavage of mica originates from the weak bonds between the potassium ions and the two adjacent aluminosilicate layers, and it has been claimed that upon cleavage the atomic structure of the aluminosilicate layers is undisturbed while the potassium layer is disrupted [15]. Therefore, a cleaved mica surface exhibits a hexagonal arrangement of $\mathrm{Si}$ (partly $\mathrm{Al}$ ) and $\mathrm{O}$ atoms, as illustrated in figure 1(b); however, this surface can be expected to be partly covered by potassium ions from the cationic layer.

Based on this structure, the surface preparation of mica appears to be straightforward, suggesting that highly resolved images revealing atomic structures should be possible without further surface treatment. Indeed, a number of studies exists, demonstrating lattice resolution, namely the regular arrangement of hexagonal $\mathrm{SiO}_{4}$ rings [16-23]. Such results (revealing structural periodicity but no individual atomicscale defects) can quite easily be obtained with contactmode atomic force microscopy operated either in ambient atmosphere [16, 18, 19] or in a liquid environment [20, 21]. Therefore, mica is often used as a standard for calibration and performance testing of commercial force microscopes operated in the contact mode. Recently, imaging with atomic-scale resolution of the lattice and atomic defects using non-contact atomic force microscopy (NC-AFM) in liquid environment has been demonstrated [22].

The initial intention of the present study was to prepare and to measure the mica surface under the controlled conditions of an ultra-high vacuum (UHV) to reveal the surface atomic structure as we have demonstrated it for other dielectric crystalline surfaces [24]. Upon cleavage in UHV, however, the mica surface is found to be highly charged, effectively prohibiting NC-AFM imaging of any reasonable resolution. This peculiarity stems from the strong interaction between inhomogeneously distributed surface charges and the tip of the force microscope, introducing fluctuating forces during scanning that outbalance by orders of magnitude the subtle force contrast features representing nanoscopic and atomicscale surface structures. Air-cleaved surfaces measured under UHV conditions, in contrast, reveal far less surface charge and can readily be imaged with NC-AFM.

In the present study, we explore the surface structure and limits of resolution that can be obtained when investigating mica sheets prepared under the practically relevant conditions of cleavage in air while imaging is performed under UHV conditions. On such a surface we find a large number of small particles covering the surface, but also larger, more regularly shaped islands. These results strengthen previous speculations proposing that the islands originate from a reaction of residual surface potassium ions with carbonaceous gases and water, forming potassium carbonate crystallites. Most importantly, we demonstrate that this contamination layer is not removed using standard UHV cleaning procedures.

\section{Experimental procedures}

Experiments are performed in a UHV system with a base pressure below $10^{-10}$ mbar consisting of a preparation chamber with a home-built UHV cleavage facility and a measurement chamber equipped with a commercial dynamic atomic force microscope (UHV 750 from RHK, Troy, Michigan, USA) that is operated in the non-contact mode as described in our earlier studies [25]. As force sensors, we use p-doped silicon cantilevers (PPP-QFMR from Nanosensors, Neuchâtel, Switzerland) with a resonance frequency of about $70 \mathrm{kHz}$, a spring constant of about $2.8 \mathrm{~N} \mathrm{~m}^{-1}$, and a $Q$ value above 80000 in UHV. The cantilever oscillation amplitude is kept constant at a level of $10-15 \mathrm{~nm}$. All tips are covered by a native oxide layer when purchased. We use these tips without any further treatment; however, we perform a bake-out at $400 \mathrm{~K}$ in UHV to remove volatile contaminants prior to AFM imaging. When approaching the surface with the oscillating cantilever, the tip-surface interaction results in a change in the resonance frequency, commonly referred to as detuning. Two operation modes are possible, namely the constant height mode and the constant detuning mode [26]. It turned out that only the constant height imaging mode is capable of providing highestresolution images of the nanoscopic details that we observe on the mica surface. This mode can, however, only be used when scanning on fairly flat terraces. Images recorded in the constant height mode directly provide the detuning signal $\Delta f$ but do not provide any topographic information. In contrast, for imaging high clusters and crystallites, the constant detuning mode is used. In this mode, the detuning signal is kept constant by adjusting the tip-surface distance, and the error signal of the regulation loop provides the topography signal $z$.

For best imaging contrast on air-cleaved surfaces, the tipsurface electrostatic interaction is minimized by a variable sample bias voltage of typically -2.5 to $+2.5 \mathrm{~V}$ applied at the metallic sample support [27]. For the UHV-cleaved surfaces, however, a bias voltage of more than $-130 \mathrm{~V}$ might be needed to compensate the electrostatic tip-sample interaction. Low-energy electron diffraction (LEED) data were obtained in another UHV chamber equipped with a fourgrid diffractometer (RVL 8-120 M2 from Princeton Research Instruments, Princeton, New Jersey, USA).

The mica samples originated from Plano GmbH (Wetzlar, Germany) and are of best available quality (Hi-Grade Quality). In order to obtain very clean mica samples and for ease of sample handling, the initial mica sheet was repeatedly cut and cleaved (every cut was followed by a cleave) until the specimen 
would fit into the sample holder. We cleave samples by scoring with a knife not by attaching and removing adhesive tape, thereby avoiding contamination that might originate from the tape. The final cleavage for ex situ preparation is performed with mica already mounted on the sample holder in order to avoid contamination from the insertion into the sample holder. For UHV cleavage of mica samples, we use a home-built cleavage facility consisting of a knife attached to a wobble stick.

Prior to NC-AFM experiments, air-cleaved mica samples are degassed at temperatures ranging from 500 to $560 \mathrm{~K}$ in an attempt to remove adsorbed water and other airborne contaminants, following recipes reported in the literature [6-9, 14]. Prior to LEED experiments, the air-cleaved mica samples are treated similarly with degassing temperatures varying from 400 to $850 \mathrm{~K}$. The sample temperature is measured by a type-K thermocouple attached to the sample holder close to the mica sample. To explore the preparation conditions, we have systematically varied two major parameters, namely the degassing temperature and the degassing period, and relate these to the resulting surface quality. As the optimum we found the abovementioned temperatures and a degassing period of about two hours, which is similar to parameters reported in the literature $[6-9,14]$.

\section{Results and discussion}

When cleaving mica in UHV, the surface potential is typically $-80 \mathrm{~V}$, and we occasionally find values above $-130 \mathrm{~V}$. However, even upon applying an overall compensating voltage between the tip and the sample support, imaging the surface at such high potentials is not possible as charge is distributed inhomogeneously and spatially fluctuating electrostatic forces dominate the AFM contrast. We tried to remove the charges by annealing after cleavage; however, even annealing at $560 \mathrm{~K}$ for $12 \mathrm{~h}$ did not reduce the surface potential significantly. As another strategy for removing surface charges, we exposed UHV-cleaved samples to air. It was found that UHV-cleaved samples had to be exposed to air for a couple of minutes to reduce the potential to values within the range of -2.5 to $+2.5 \mathrm{~V}$ which usually allows high-resolution imaging. The surface morphology of UHV-cleaved mica samples that are exposed to air after cleavage is similar to the morphology of surfaces prepared by cleavage in air.

It has been suggested that the interlayer potassium ions are statistically shared between the two freshly cleaved mica sheets after cleavage and, therefore, any freshly cleaved mica surfaces is expected to be covered with half a monolayer of potassium ions that are, however, not ordered in a specific structure or reconstruction [15]. Therefore, we ascribe the observed strong charging of UHV-cleaved mica surfaces to the presence of potassium ions. Several methods for neutralization or removal of the residual surface potassium ions have been suggested in the literature, including neutralization and sputtering of potassium with $1 \mathrm{keV}$ electrons [3], and sublimation of potassium by substrate heating $(600-900 \mathrm{~K})$ [3, 28] or laser irradiation [29]. However, none of these experiments yielded convincing evidence that the mica surface can be neutralized
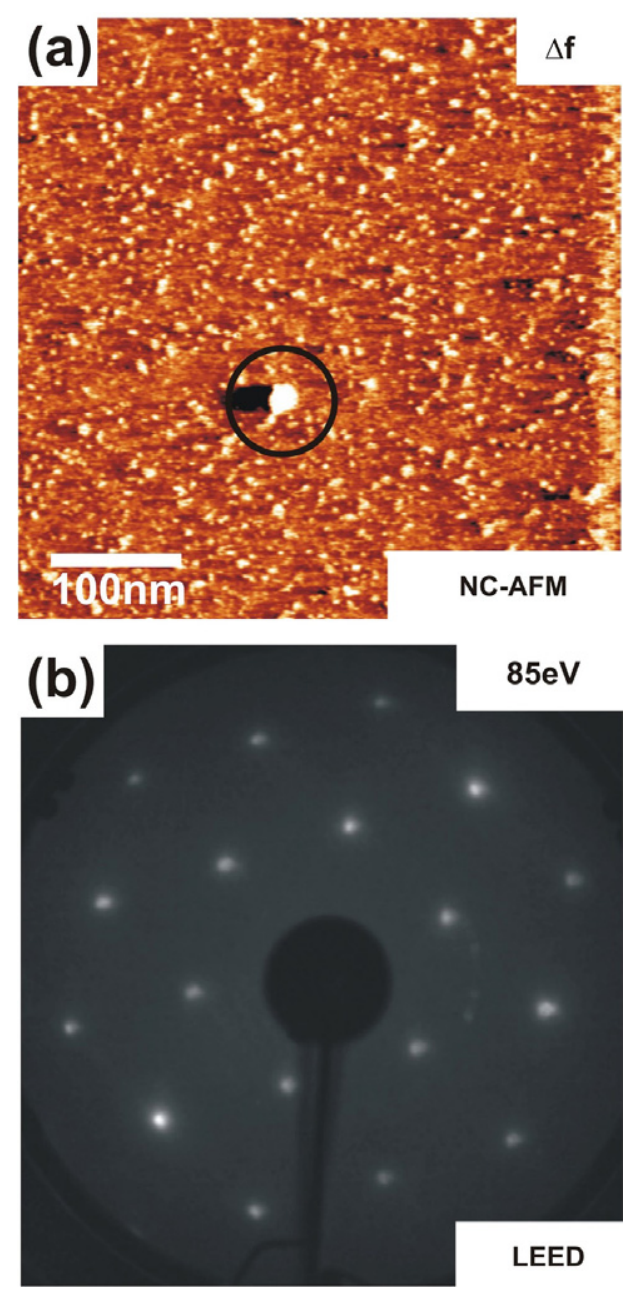

Figure 2. NC-AFM image and LEED pattern of air-cleaved muscovite mica surfaces after degassing in UHV. (a) NC-AFM image recorded in the constant height mode with an average detuning of $-69 \mathrm{~Hz}$ and a bias voltage of $-0.92 \mathrm{~V}$. A high density of small particles and one larger structure (marked by a circle) are observed. The dark region to the left of this larger structure is an experimental artefact due to imaging with a topography loop with a very small loop gain. This sample was degassed in UHV at $560 \mathrm{~K}$ for $2 \mathrm{~h}$. (b) LEED pattern obtained at an electron energy of $85 \mathrm{eV}$ after degassing in UHV at $850 \mathrm{~K}$ for several hours. Degassing at $400 \mathrm{~K}$ for several hours reveals an identical LEED pattern.

in one of these ways without being damaged. Therefore, we focus here entirely on the investigation of the air-cleaved mica surface and leave efforts to prepare a perfect UHV-cleaved mica surface to future studies.

The typical surface morphology of an air-cleaved mica surface is presented in the image shown in figure 2(a). Air-cleaved mica surfaces always exhibit a high density of small particles and a small number of larger structures. We investigated the influence of degassing on the surface cleanliness by a systematic variation of degassing temperature and degassing period. Temperatures were varied from 350 to $560 \mathrm{~K}$, and the degassing period was increased up to $18 \mathrm{~h}$. However, none of these modifications led to a removal of the particles, and it was not possible to obtain a clean surface locally exhibiting atomic structure. To check 
whether the surface below the particles is atomically well ordered, we performed low-energy electron diffraction (LEED) measurements that do not probe the local atomic structure but an average structure sampled over a surface area with a diameter of $1 \mathrm{~mm}$ and a depth of about $1 \mathrm{~nm}$. As shown in figure 2(b), the LEED pattern reveals bright and welldefined spots in a hexagonal arrangement, corresponding to the hexagonal atomic surface structure of mica. Also, the LEED pattern was found to be independent of degassing temperature when varied from 400 to $850 \mathrm{~K}$. This result suggests that the surface underneath the small particles is indeed the unperturbed mica cleavage plane.

An analysis of the small particles yields that their density is independent of the annealing temperature and duration. The density of the small particles in figure 2(a) amounts to 4500 particles per $\mu \mathrm{m}^{2}$. The apparent lateral size of particles ranges from 1 to $5 \mathrm{~nm}$. To achieve higher resolution on and in between the particles, we performed further NC-AFM experiments. Figures 3(a) and (b) present high-resolution NC-AFM images obtained on another mica surface with a different AFM tip. Besides reducing the scan size, we reduced the tip-sample distance. Under such imaging conditions, the particles are not imaged as protrusions but as apparent depressions with a bright rim. This is due to a peculiarity in AFM contrast formation, indicating a transition from purely attractive to the onset of repulsive interaction forces between the tip and the particles that is discussed in detail elsewhere [33]. The shape and position of the particles are robust against this close approach by the tip, indicating that they are firmly anchored on the surface. Although the regions in between the particles appear to be atomically flat (see figure 3(b)), it is not possible to reveal any atomic structure as atomic contrast features are covered by fluctuations due to the nanoscale roughness of the surface. The particle density in figures 3(a) and (b) amounts to 20800 and 38500 particles per $\mu \mathrm{m}^{2}$, respectively. These density values indicate that only a fraction of the particles are seen in the large-scale images, illustrating that a precise determination of particle densities may be a delicate question of choosing the right frame size.

To investigate larger structures like the one circled in figure 2(a) with respect to their topography and morphology, we switched from imaging in the constant height mode to the constant detuning mode. This allows us to determine the height of the larger structures, which varies between 0.6 and $5.0 \mathrm{~nm}$. Larger structures often appear to be rather regular with respect to both a regular shape as well as a step structure with atomically flat terraces. A typical example for an island of $1.7 \mathrm{~nm}$ height is shown in figure 4(a), while islands with a height of $0.6 \mathrm{~nm}$ are most abundant. In figure 4(b) we see an island of $5 \mathrm{~nm}$ height, exhibiting a distinct step structure. The zoom into the area shown in figure 4(c) exhibits steps of 0.3 and $0.6 \mathrm{~nm}$ height separating atomically flat terraces.

\section{Conclusion}

On the basis of our observations and models available in the literature, we have developed a model for the formation of small particles and large islands on air-cleaved mica. The
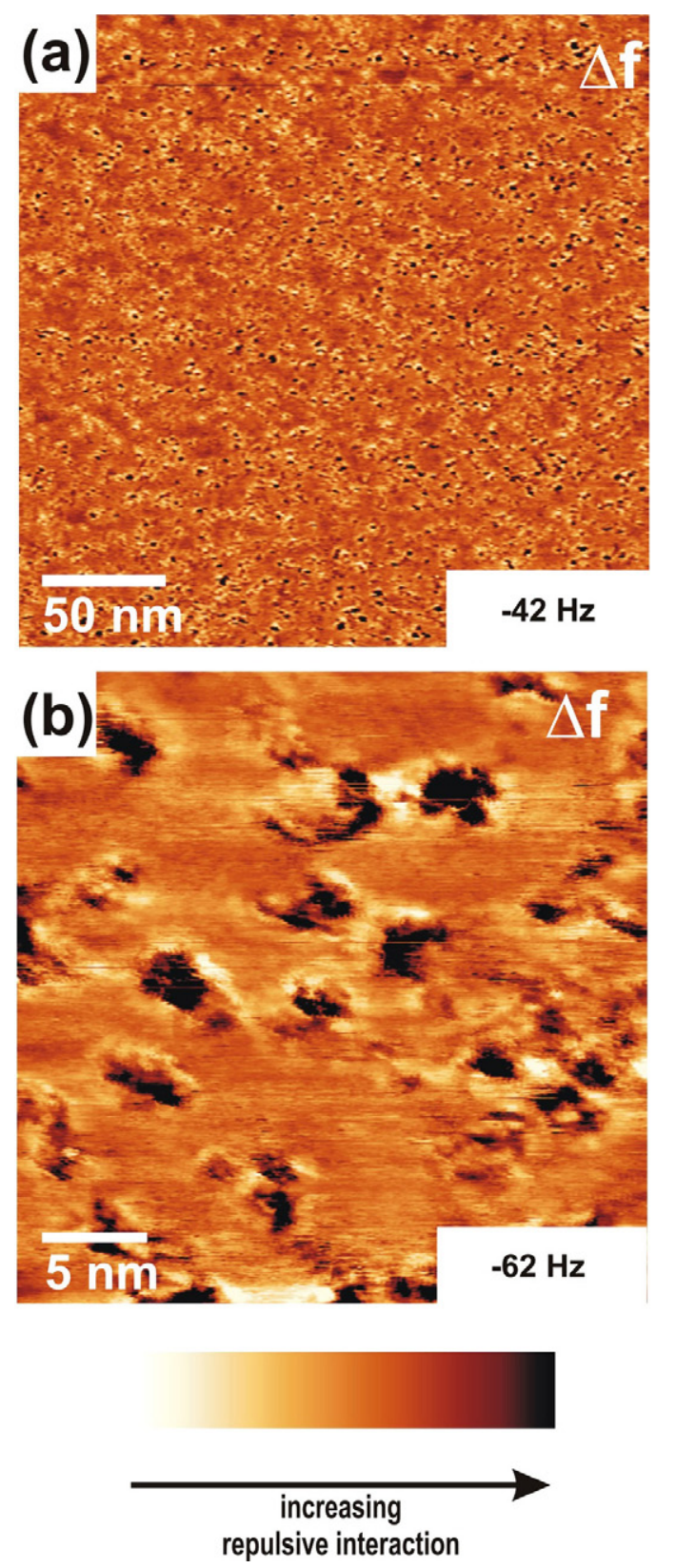

Figure 3. Air-cleaved muscovite mica surface after degassing in UHV at $473 \mathrm{~K}$ for $2 \mathrm{~h}$. (a) NC-AFM image recorded in the constant height mode with an average detuning of $-42 \mathrm{~Hz}$. A high density of small particles is observed. (b) Zoom into the area combined with reducing the tip-sample distance, resulting in a detuning of $-62 \mathrm{~Hz}$. In both images, the particles are imaged as depressions due to a contrast inversion, which can be ascribed to the onset of repulsive interaction. (Note that the detuning values given here cannot be compared with the one from figure 2(a) as different tips were used.)

key assumption is that, immediately after cleavage in air, the residual potassium ions react strongly with constituents of the surrounding atmosphere. It has been proposed that a reaction of carbonaceous gases is specifically involved, yielding a carbon-containing contamination layer $[3,28,30]$. This layer has been measured with the surface force apparatus, yielding a layer thickness of about $0.3-0.4 \mathrm{~nm}[12,31]$. Furthermore, it has been reported that this contamination layer is not created 

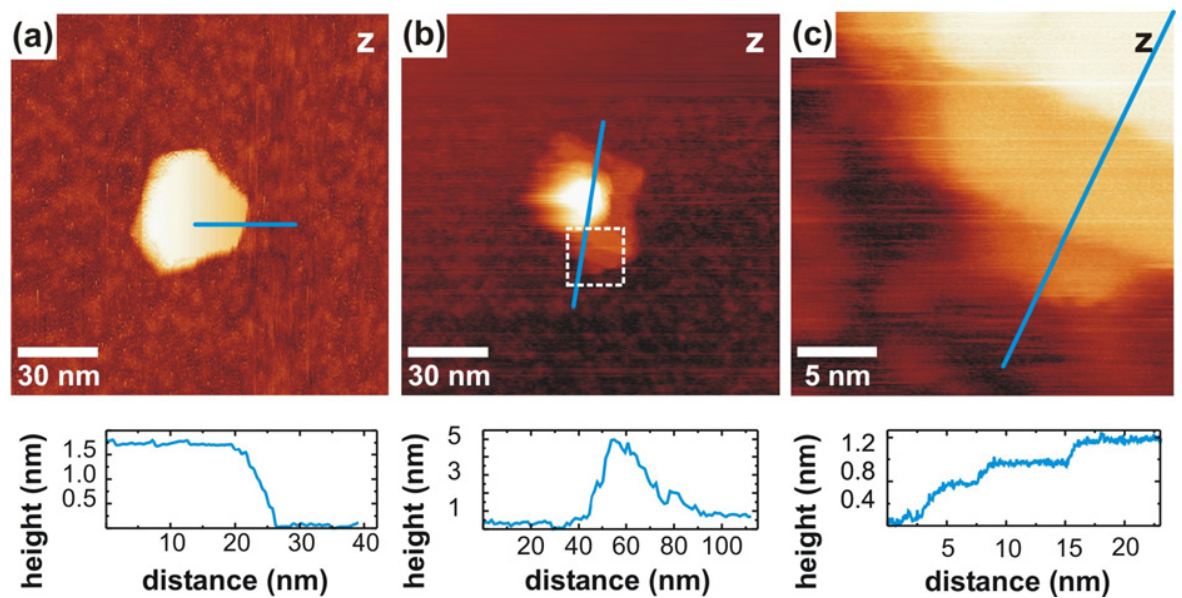

Figure 4. Large regularly shaped islands on air-cleaved mica surfaces after degassing in UHV at $473 \mathrm{~K}$ for $14 \mathrm{~h}$ recorded in the constant detuning mode. NC-AFM images recorded in the constant detuning mode. The profiles shown below the images are cross-sections taken along the indicated lines. (a) Island of $1.7 \mathrm{~nm}$ height with regular shape. Image taken at a detuning of $-7.3 \mathrm{~Hz}$ and a bias voltage of $0 \mathrm{~V}$. (b) Island of $5 \mathrm{~nm}$ height. The extended bright lobes in the centre of this structure are a scanning artefact. Image taken at a detuning of $-4.0 \mathrm{~Hz}$ and a bias voltage of $-1.3 \mathrm{~V}$. (c) Zoom into the structure marked by the square in frame (b) revealing three terraces. Image taken at a detuning of $-22.1 \mathrm{~Hz}$ and a bias voltage of $0 \mathrm{~V}$.

by simple adsorption of $\mathrm{CO}$ or $\mathrm{CO}_{2}$, but only when water is present and acting as a binding agent $[3,30]$. It has, therefore, been suggested that the contamination layer originates from a reaction of $\mathrm{CO}_{2}$ with water and potassium ions, resulting in potassium carbonate [12]. This model well explains why air-cleaved surfaces show a strongly reduced surface charge compared to the UHV-cleaved surface, as the surface reaction readily compensates the potassium excess charge.

Beside the contamination layer, the high-resolution NCAFM images reveal regularly shaped islands of about $30 \mathrm{~nm}$ size. The regular shape of these islands resembles the shape of the micrometre-sized structures observed before with scanning electron microscopy [11]. These larger structures have been ascribed to potassium carbonate crystallites that grow from the contaminants during drying [12]. We, therefore, assume that the regularly shaped islands observed in our study consist of potassium carbonate crystallites that grow from dissolved $\mathrm{CO}_{2}$ and potassium ions within a water film on the surface. As the crystal growth takes place within the water film, the size of the crystallites is likely to depend on both the amount of water on the surface and the details of the drying procedure. Due to the fact that potassium carbonate has a good solubility in aqueous solution and that adsorbed layers are easily removed upon direct contact with a scanning tip [32], it can well be understood why neither contact-mode AFM nor NC-AFM when performed in an ambient or liquid environment revealed contaminated samples or crystallite growth.

As the final most important result, we have shown that standard cleaning procedures that are commonly applied to air-cleaved mica surfaces when using them as a substrate in UHV fail to remove the adsorbed layer of contaminants. Even prolonged heating at elevated temperatures under UHV conditions does not result in an atomically flat substrate surface. In future studies we will explore strategies such as ion exchange in dilute acid solutions [11] for removing the surface charges to evaluate whether these methods are also applicable for transforming mica into a UHV-compatible substrate.

\section{Acknowledgments}

Financial support from the EU FP7 integrated project PicoInside and the EU Marie Curie action NEED is gratefully acknowledged. We are indebted to R Bechstein, P Rahe and $\mathrm{T}$ Dienel for most stimulating discussions.

\section{References}

[1] Hochella M F 1990 Rev. Mineral. 2387

[2] Douglas T 2003 Science 2991192

[3] Poppa H and Elliot A G 1971 Surf. Sci. 24149

[4] Hansma P K et al 1994 Appl. Phys. Lett. 641738

[5] Cai L T, Tabata H and Kawai T 2000 Appl. Phys. Lett. 773105

[6] Ferrero S, Piednoir A and Henry C R 2001 Nano Lett. 1227

[7] Derose J A, Thundat T, Nagahara L A and Lindsay S M 1991 Surf. Sci. 256102

[8] Proehl H, Nitsche R, Dienel T, Leo K and Fritz T 2005 Phys. Rev. B 71165207

[9] Proehl H, Dienel T, Nitsche R and Fritz T 2004 Phys. Rev. Lett. 93097403

[10] Israelachvili J N, Alcantar N A, Maeda N, Mates T E and Ruths M 2004 Langmuir 203616

[11] Christenson H K and Israelachvili J N 1987 J. Colloid Interface Sci. 117576

[12] Christenson H K 1993 J. Phys. Chem. 9712034

[13] Ohnishi S, Hato M, Tamada K and Christenson H K 1999 Langmuir $\mathbf{1 5} 3312$

[14] Lee S M and Krim J 2005 Thin Solid Films 489325

[15] Muller K and Chang C C 1969 Surf. Sci. 1439

[16] Sharp T G, Oden P I and Buseck P R 1993 Surf. Sci. 284 L405

[17] Fukuma T, Kilpatrick J I and Jarvis S P 2006 Rev. Sci. Instrum. 77123703

[18] Erlandsson R, Hadziioannou G, Mate C M, McClelland G M and Chiang S 1988 J. Chem. Phys. 895190

[19] Baba M, Kakitani S, Ishii H and Okuno T 1997 Chem. Phys. 22123

[20] Kuwahara Y 1999 Phys. Chem. Minerals 26198 
[21] Kuwahara Y 2001 Phys. Chem. Minerals 281

[22] Fukuma T, Kobayashi K, Matsushige K and Yamada H 2005 Appl. Phys. Lett. 87034101

[23] Fukuma T and Jarvis S P 2006 Rev. Sci. Instrum. 77043701

[24] Reichling M and Barth C 1999 Phys. Rev. Lett. 83768

[25] Hirth S, Ostendorf F and Reichling M 2006 Nanotechnology $17 \mathrm{~S} 148$

[26] Gritschneder S, Namai Y, Iwasawa Y and Reichling M 2005 Nanotechnology 16 S41

[27] Gritschneder S and Reichling M 2007 Nanotechnology 18044024
[28] Dowsett M G, King R M and Parker E H C 1977 J. Vac. Sci. Technol. 14711

[29] Gerlach R, Polanski G and Rubahn H G 1996 Surf. Sci. 352485

[30] Bhattacharyya K G 1989 Langmuir 51155

[31] Israelachvili J N and Adams G E 1978 J. Chem. Soc. Faraday Trans. 74975

[32] Hu J, Xiao X D, Ogletree D F and Salmeron M 1995 Surf. Sci. 327358

[33] Rahe P, Bechstein B, Schütte J, Ostendorf F and Kühnle A 2008 Phys. Rev. B 77195410 